\title{
Synthesis and Alignment of Silver Nanorods and Nanowires and the \\ Formation of Pt, Pd, and Core/Shell Structures by Galvanic Exchange \\ Directly on Surfaces
}

\author{
Grzegorz W. Sławiński and Francis P. Zamborini*
}

\section{SUPPORTING INFORMATION}

Figure S1 shows the UV-Vis spectra of growth solutions, where the left plot shows all spectra as in Figure 1B in the main paper and the right plot is an expanded plot of the low absorbance spectra (all pH values other than 11.9).

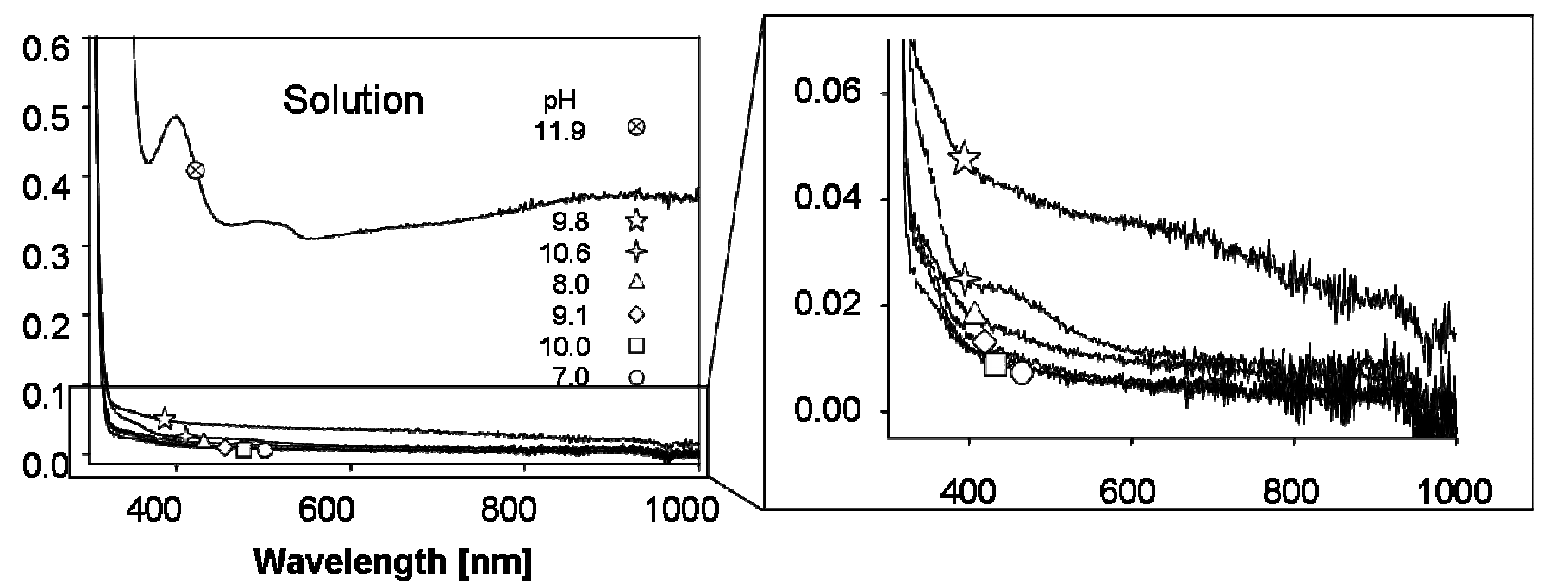

Figure S1. Original UV-Vis spectra of Ag growth solutions (left plot) as in Figure 1B of the main paper and expanded plot of the low absorbance spectra other than $\mathrm{pH} 11.9$ (right plot).

Table S1 shows Ag nanostructure coverage measurements (in number of particles per $\mu \mathrm{m}^{2}$ ) obtained from at least 10 different regions of at least three different samples as a function of $\mathrm{pH}$ of the growth solution used to grow the Ag nanostructures. The table shows the normal growth procedure and procedure used for growing aligned $\mathrm{Ag}$ NRs/NWs at $\mathrm{pH} 10.6$, labeled as A and B, respectively. We calculated the average coverage and standard deviation for 


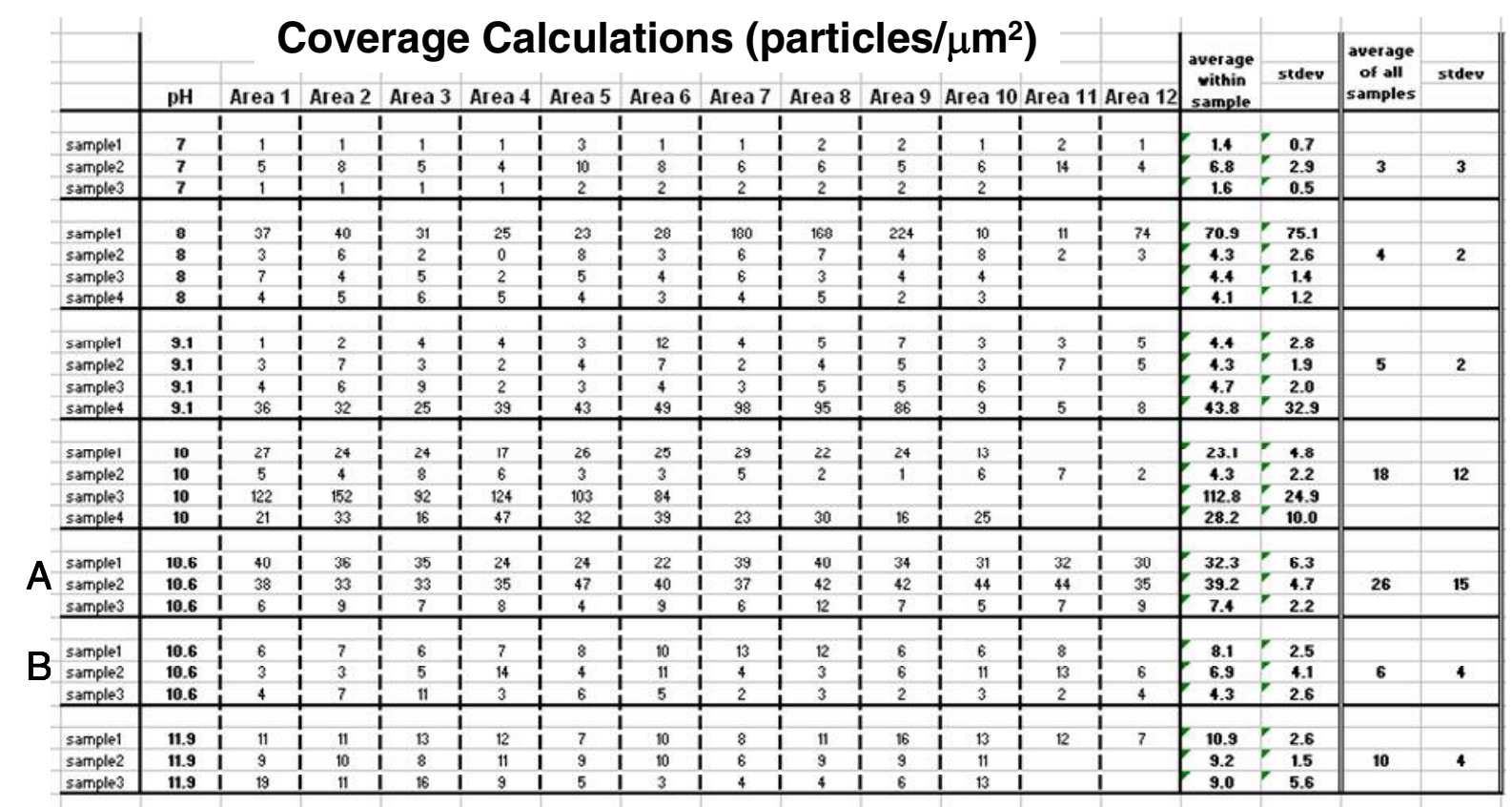

Table S1. The coverage of Ag nanostructures in particles per $\mu \mathrm{m}^{2}$ for at least 10 different areas of at least 3 different samples as a function of the $\mathrm{pH}$ of the growth solution. A and $\mathrm{B}$ for $\mathrm{pH} 10.6$ represent $\mathrm{Ag}$ nanostructures grown by the normal method and aligned Ag nanostructures, respectively. Values with * were not counted for statistics (explanation in text).

different areas within the same sample and for all areas of different samples as shown in the table. Figure 3 in the paper shows the average coverage and standard deviations considering all areas of all samples at a given $\mathrm{pH}$ value as shown in this table. We considered one sample at $\mathrm{pH}$ 8.0, $\mathrm{pH} 9.1$, and $\mathrm{pH} 10.0$ as an outlier (marked with a *) and did not count it in the overall average and standard deviation shown here and in Figure 3. These samples had an exceptionally large number of extremely small particles on the surface, leading to larger coverages compared to the other three samples measured for that particular $\mathrm{pH}$. Not including these three samples, the deviation in coverage within the same sample from area to area was generally $20-40 \%$, while sample-to-sample deviation was generally greater than 50\%. Although there is a high deviation in coverage from area to area, the trend in $\mathrm{pH}$ and the synthesis of $\mathrm{Ag}$ NRs and NWs at pH 10.6 was highly reproducible. 
The SEM images in Figure S2 give an example of the deviation in coverage and in Ag particle size observed for 3 different areas of 3 different samples synthesized at $\mathrm{pH}$ 10.0. Most samples are more uniform than Figure S2 indicates, but this is an example of what sometimes occurs. Figure S2A, S2B, and S2C, respectively, shows representative areas characterized as low, medium, and high coverage. The coverage in number of nanostructures per $\mu \mathrm{m}^{2}$ are $<20,20-100$, and $>100$, respectively. The high density areas (Figure S1C) were not included in the statistics because they occurred very rarely, are difficult to measure due to the small size, and, although they do not represent the majority of the surface, they have a large impact on the statistics. As expected for seed-mediated growth, the average particle size decreases as the density of structures increases. This is expected if we assume an equal amount of Ag deposits on a given area during a certain time. With more seed particles, if the Ag is deposited evenly over the particles, each particle will grow less when compared to an area with a smaller number of seed particles, where the same amount of $\mathrm{Ag}$ will be distributed over fewer seed particles leading to more growth per particle.

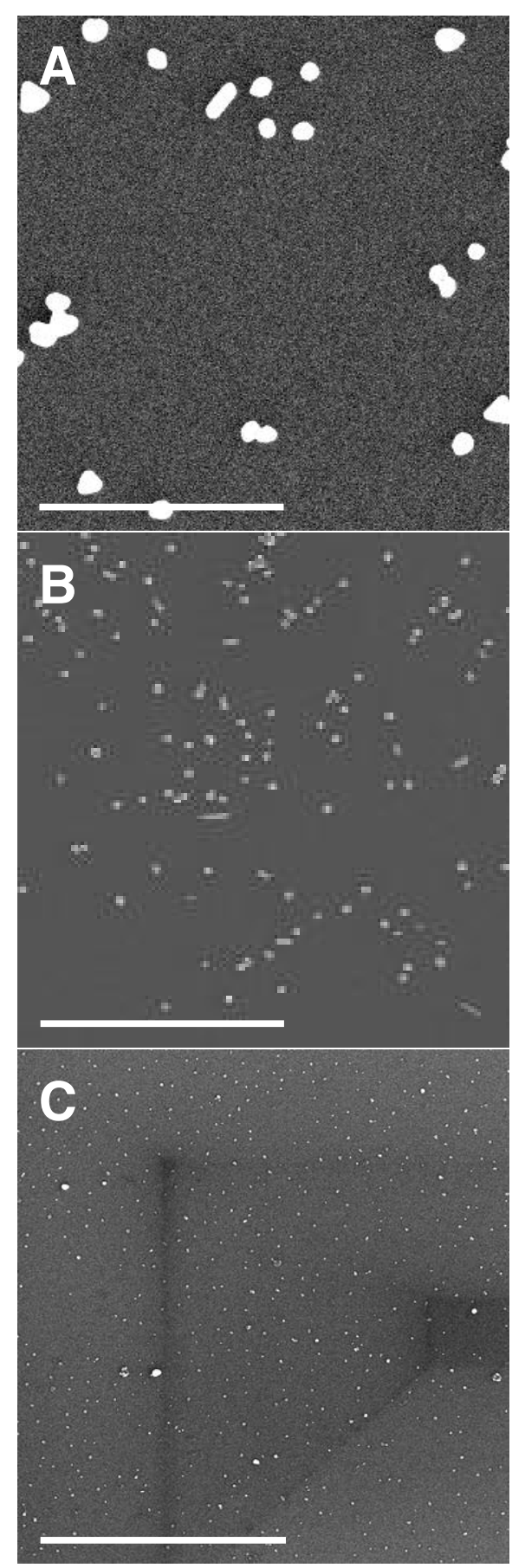

Figure S2. SEM images of three different areas of $\mathrm{Ag}$ nanostructures grown at $\mathrm{pH}$ 10.0. The coverage of nanostructures is $(\mathrm{A})<20 / \mu \mathrm{m}^{2}$ "low", (B) $20-100 / \mu \mathrm{m}^{2}$ "medium", and (C) $>$ $100 / \mu \mathrm{m}^{2}$ "high". Scale bar is $1 \mu \mathrm{m}$ in all images. 
To verify that the Ag nanostructures grow by seed-mediated growth, we placed bare glass and MPTMS-functionalized glass samples (without seed) into Ag growth solution at $\mathrm{pH} 10.6$ and monitored them by UV-vis spectroscopy. Figure S3, plots B and $\mathrm{C}$, shows the UV-vis spectrum of the bare and MPTMS-functionalized glass slides, respectively, after being in $\mathrm{pH}$ 10.6 Ag growth solution for 30 min. Plot A shows the spectrum of an MPTMSfunctionalized glass slide that

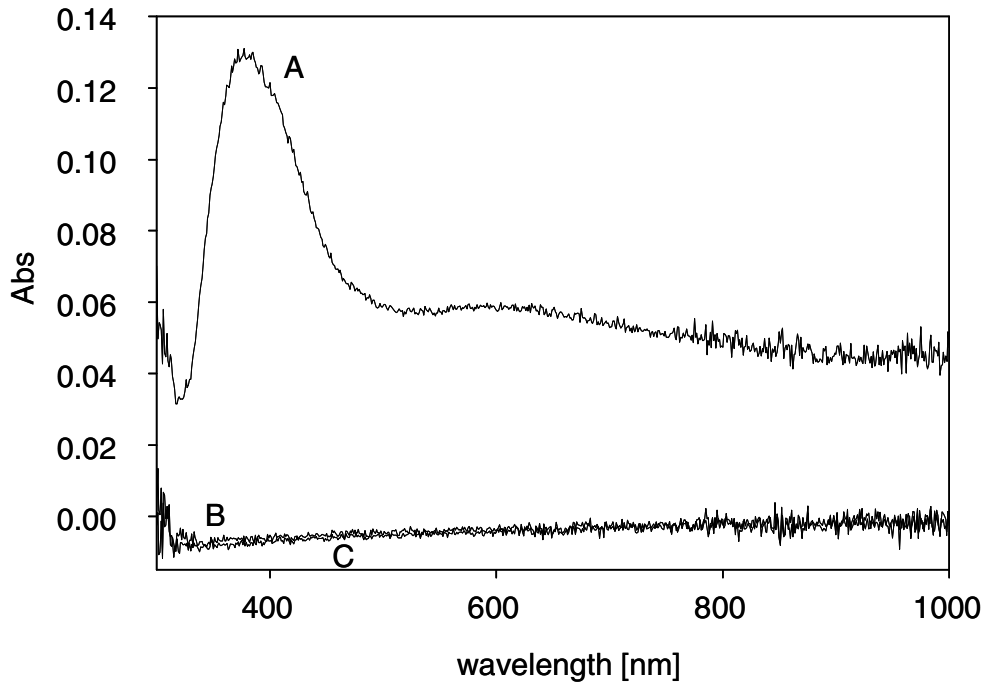

Figure S3. UV-Vis spectra of (A) Glass/MPTMS/Au NP, (B) Glass/MPTMS, and (C) Bare Glass samples placed in Ag growth solution at $\mathrm{pH} 10.6$ for $30 \mathrm{~min}$.

was placed in Au seed nanoparticles for 20 min and then $\mathrm{pH} 10.6 \mathrm{Ag}$ growth solution for $30 \mathrm{~min}$. Plot A shows an Ag surface Plasmon band at 400 nm with a peak absorbance of 0.13, consistent with the growth of Ag nanostructures on the surface. In contrast, plots B and C show no surface plasmon band and no absorbance in the visible region. This shows that the Au seed nanoparticles are necessary for Ag growth to occur, which is consistent with a seed-mediated growth process.

Frames A, B, and C of Figure $\mathrm{S} 4$ show $\mathrm{SEM}$ images of $\mathrm{Si} / \mathrm{SiO}_{\mathrm{x}}$ substrates prepared identically to the three samples prepared on glass to obtain the UV-vis spectra shown as plots A, $\mathrm{B}$, and $\mathrm{C}$ of Figure S3. Frame A shows a considerable amount of Ag nanostructures grown on the surface, including the presence of high aspect ratio Ag nanorods and nanowires. Frames B and $\mathrm{C}$ show very few particles on the surface. These images are consistent with the absorbance 
values for the same samples prepared on glass (Figure S3)

and with a seed-mediated growth process occurring since no nanostructures grew on the surfaces without Au nanoparticle seeds present.

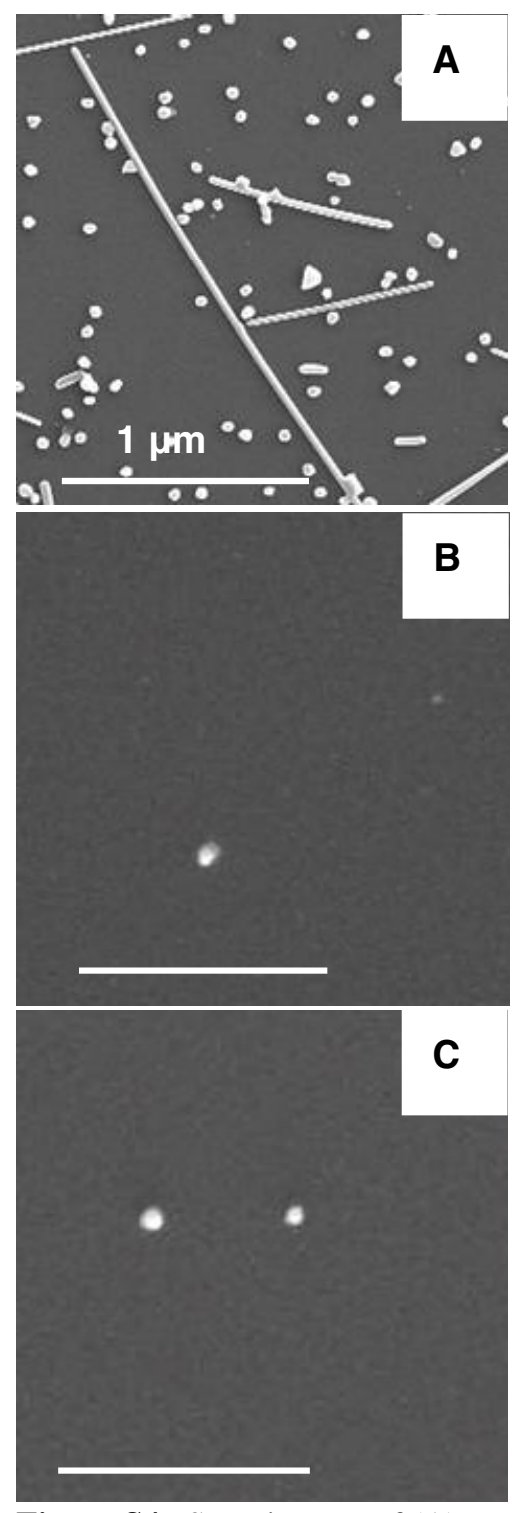

Figure S4. SEM images of (A) $\mathrm{Si} / \mathrm{SiO}_{\mathrm{x}} / \mathrm{MPTMS} / \mathrm{Au} \mathrm{NP}$, (B) $\mathrm{Si} / \mathrm{SiO}_{\mathrm{x}} / \mathrm{MPTMS}$, and (C) Bare $\mathrm{Si} / \mathrm{SiO}_{\mathrm{x}}$ samples placed in $\mathrm{Ag}$ growth solution at $\mathrm{pH} 10.6$ for 30 min. Scale bar is $1 \mu \mathrm{m}$ in all images. 